\title{
Molgenis-impute: imputation pipeline in a box
}

Alexandros Kanterakis ${ }^{*}{ }^{\dagger}$, Patrick Deelen ${ }^{\dagger}$, Freerk van Dijk, Heorhiy Byelas, Martijn Dijkstra and Morris A Swertz

\begin{abstract}
Background: Genotype imputation is an important procedure in current genomic analysis such as genome-wide association studies, meta-analyses and fine mapping. Although high quality tools are available that perform the steps of this process, considerable effort and expertise is required to set up and run a best practice imputation pipeline, particularly for larger genotype datasets, where imputation has to scale out in parallel on computer clusters.

Results: Here we present MOLGENIS-impute, an 'imputation in a box' solution that seamlessly and transparently automates the set up and running of all the steps of the imputation process. These steps include genome build liftover (liftovering), genotype phasing with SHAPEIT2, quality control, sample and chromosomal chunking/merging, and imputation with IMPUTE2. MOLGENIS-impute builds on MOLGENIS-compute, a simple pipeline management platform for submission and monitoring of bioinformatics tasks in High Performance Computing (HPC) environments like local/cloud servers, clusters and grids. All the required tools, data and scripts are downloaded and installed in a single step. Researchers with diverse backgrounds and expertise have tested MOLGENIS-impute on different locations and imputed over 30,000 samples so far using the 1,000 Genomes Project and new Genome of the Netherlands data as the imputation reference. The tests have been performed on PBS/SGE clusters, cloud VMs and in a grid HPC environment.

Conclusions: MOLGENIS-impute gives priority to the ease of setting up, configuring and running an imputation. It has minimal dependencies and wraps the pipeline in a simple command line interface, without sacrificing flexibility to adapt or limiting the options of underlying imputation tools. It does not require knowledge of a workflow system or programming, and is targeted at researchers who just want to apply best practices in imputation via simple commands. It is built on the MOLGENIS compute workflow framework to enable customization with additional computational steps or it can be included in other bioinformatics pipelines. It is available as open source from: https://github. $\mathrm{com} / \mathrm{molgenis/molgenis-imputation.}$
\end{abstract}

Keywords: Imputation, Genotyping, GWAS

\section{Background}

Genotype imputation uses densely typed reference haplotypes to infer untyped genotypes [1]. The resulting imputed datasets are commonly used in meta-analyses to gain statistical power or for the fine-mapping of association signals [2]. Modern imputation methods enable inference of many types of genetic variation, including

\footnotetext{
*Correspondence: alexandros.kanterakis@gmail.com

${ }^{\dagger}$ Alexandros Kanterakis and Patrick Deelen contributed equally to this work

Department of Genetics, Genomics Coordination Center, University Medical Center Groningen and University of Groningen, Genetics, UMCG, PO Box 30 001, 9700 RB Groningen, The Netherlands
}

single nucleotide polymorphisms (SNPs), insertions and deletions [3].

Imputation has been widely adopted as it has led to the identification of additional associations [4] and has allowed combination studies from different genotyping platforms [5, 6] contributing to a meta-analysis [7]. Another benefit is the fine mapping of association signals: since detected regions are not usually located in a functional region but due to linkage disequilibrium (LD) they are highly associated with the true causal variant that might not have been assayed at all. Finally, it has been suggested [8] that by using imputation we can enrich rare variants with large effect that contribute 
significantly to the 'missing heritability' of complex traits such as lipid profiles.

Today, there are high quality tools that perform imputation, including Minimac [9], BEAGLE [5] and IMPUTE2 [2]. Many studies have evaluated their performance regarding parameters such as the genotype platform of the imputed study, the number of SNPs, ethnicity of the samples, reference panels, LD structure, allele frequency of variants, improvement of statistical power in genome-wide association studies (GWAS), and enhancement in the identification of causal variants [10-14].

The general consensus from these comparisons is that these three imputation tools exhibit a similar performance in terms of the estimated correlation between the imputed and true genotypes. Although all the tools could be easily added, we decided to include IMPUTE2 because of previous experience in our team, reported marginal benefits in terms of unrelated reference panels [14], and execution times when using prephased data [15]. The phasing software that we selected was SHAPEIT [16], mainly because it integrates nicely with IMPUTE2 and is highly recommended by the authors of IMPUTE2 [9]. The authors of SHAPEIT also demonstrate an improved phasing accuracy compared to other methods.

Regardless of the choice of imputation tool, considerable work has to be done before any of these can be used in a full pipeline. The reason for this is that these tools require pre- and post-processing steps such as liftovering, quality control, and splitting/merging of data in order to work effectively. Liftover is the process of changing the genomic positions of a dataset from one version of a genome assembly to another. It is very common that a study panel is aligned to a different build of a genome assembly than the reference panel. This pre-processing step is essential and precedes all genomic studies that include a study combining more than one dataset.

Existing bioinformatics pipeline management systems have a limited coverage for genotype imputation. For example, Galaxy [17] does not include imputation methods in its public database. Other workflow management systems like Taverna [18] and Ergatis [19] offer a thorough and complete toolset for describing bioinformatics workflows, but lack specific cases for genotype imputation. To our knowledge, the only bioinformatics solution relevant to imputation is GRIMP [20], but rather than performing imputation, it focuses on the analysis of the GWAS data that usually follows it. Moreover, setting up such advanced workflow systems requires more time and skill than most genetics researchers have available.

Here we present MOLGENIS-impute, a simple command line tool to run complete genotype imputation pipelines on local servers and a variety of HPC environments. This tool is for geneticists and lab bioinformaticians who simply want to perform an imputation with minimal overhead of discovering, installing and configuring tools, while ensuring best practices are followed and applying proper quality control. The steps covered are aligning markers to the same genomic reference as the reference panel (by default hg19), applying quality control to check for genomic strand inconsistencies between the study and the reference panel, phasing the study panel, splitting the study panel into multiple chromosomal and sample chunks, and merging the resulted imputed dataset. The pipeline can be executed either on a local or cloud server for smaller studies, or on an HPC cluster or grid environment for larger efforts. The solution we offer employs a fail-safe approach regarding any failures that might occur during execution. The user interacts with a simple python script via command line options. Although the required tools and commands that are necessary to execute this script are relatively common in a Linux installation, we have included a list of these tools and installation instructions in Additional file 1.

\section{Methods}

Before imputation, researchers normally spend a considerable amount of time in setting up a pipeline that includes the necessary pre- and post-processing steps, such as liftovering, quality control, and splitting/merging of data. The first main component of our approach is a single script that downloads, configures and installs all the necessary tools, data and scripts (Table 1). After careful evaluation, we selected the IMPUTE2 and SHAPEIT2 tool family for this implementation, but our pipelines can be extended to include other tools as well. The second main component is the automation of all the necessary steps. Here we use the MOLGENIS-compute package to auto-generate simple shell scripts ready for execution on a local server or for submission to a cluster or grid [21]. The only prerequisite is that the study panel should be in the default standard PLINK PED/MAP format or the binary equivalent BED/BIM/FAM [22]. Our pipeline consists of the following steps:

Step 1 is the liftovering, or the conversion of the positions of the study panel to those used by the reference panel. By default, this optional step converts from UCSC hg18 to UCSC hg19 genomic assembly. Alternatively, a user can specify a chain file in order to perform liftover between other builds of the genome. Chain files contain a mapping of the positions between two different genome assemblies. We provide a list of chain file repositories on the documentation page for MOLGENIS-impute. 
Table 1 Tools and data installed during MOLGENIS-impute set up

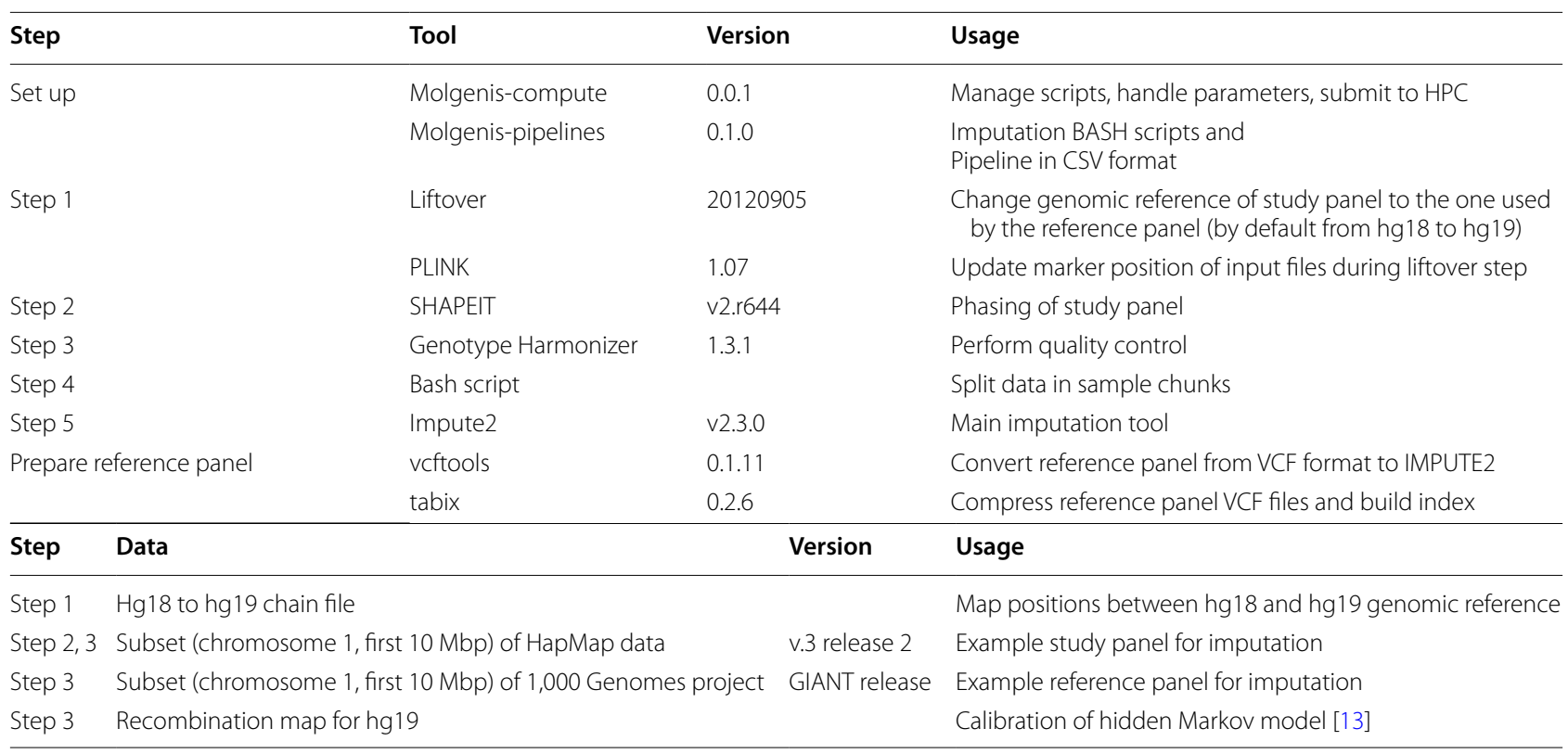

Step 2 is the phasing using SHAPEIT2, with which we infer the haplotype structure of the genotype data. Although this step is not necessary for imputation per se, it increases the imputation quality and it significantly speeds up the process. Especially when multiple imputation tasks have to be performed, phasing of the study panel only needs to be done once. This is useful when a new version of a reference panel becomes available.

Step 3 is the quality control step that guarantees that data in the study panel are aligned to the same strand as the reference panel. Alignment of $\mathrm{G} / \mathrm{C}$ and $\mathrm{A} / \mathrm{T}$ variants is performed by assessing the LD structure using Genotype Harmonizer [23]. This tool removes SNPs from the study when strand correction cannot be applied (for example, an A/T SNP in the study that exists as A/C in the reference panel). It also generates a log file of all the performed checks that includes all the removed markers. Step 4 is the splitting of the study panel into sample and chromosomal chunks. By default, each chunk contains no more than $5 \mathrm{Mbp}$ length of markers, as recommended by the IMPUTE2 software. The number of samples that each chunk has is a value between 500 and 1,000, and is devised during execution so that chunks have an approximately equal number of samples. This two-dimensional splitting is an essential step in order to handle the enormous computation that is usually required and to scale the imputation process effectively in an HPC environment.

Step 5 is the actual imputation. IMPUTE2 employs an agnostic approach regarding the population com- position of the reference panel and offers the ability to combine multiple reference panels. Upon completion of all imputation steps, we concatenate the resulting sample and chromosomal chunks for downstream analysis. IMPUTE2 generates two main results files. The first contains the posterior genotype probabilities and the second contains quality metrics per imputed marker. Merging sample chunks for the posterior genotype probabilities is trivial, since sample splitting does not affect them. Unfortunately this does not hold for the quality metrics. To overcome this, we re-compute IMPUTE2's quality metrics for the concatenated sample files. These quality metrics and the respective formulas are presented in Additional file 2.

\section{Sample study and reference panel}

The installation also contains a sample study and reference panel. The study panel is a subset of 100 samples from the HapMap project (version 2, release 3) and it contains all markers from 1 to 10 million bp in chromosome 1 . This dataset contains 4,836 markers. The sample reference panel contains all 1,092 samples of the GIANT release of the 1,000 Genomes Project reference panel. This dataset contains only the markers from 1 to 15 million bp in chromosome 1 , namely 88,650 markers.

\section{Implementation}

All computational steps are defined as templates of BASH scripts for the MOLGENIS compute system. BASH is the default shell environment in many 
modern Linux distributions, and BASH scripts are lightweight and easily embedded into external tools. The designed order of the steps in the pipeline and the input/output parameters of each step are defined in a set of CSV files [21]. MOLGENIS compute has specific mechanisms to accommodate the scripts to different backends (e.g. local, PBS, grid). Based on all of the above, MOLGENIS compute generates the actual analysis scripts. The input and output variables of these scripts are passed as BASH variables. In the header of each generated script there are definitions for the system requirements of this particular step to the specific computer cluster/grid environment. These parameters are the desired number of CPUs, amount of memory, and execution time. Additional documentation is available on https://rawgit.com/molgenis/ molgenis-compute/master/molgenis-compute-core/ README.html.

The imputation scripts belong to the MOLGENISpipelines collection, which also includes scripts and pipelines for next-generation sequencing (NGS) and GWAS analysis. They are hosted in a separate git repository: https://github.com/molgenis/molgenis-pipelines. For imputation, interested readers can check the pipeline at the following directory of the git repository: 'compute5/ Imputation_impute2'.

MOLGENIS-compute offers a mechanism to parse these template files and generate executable scripts. These scripts are adjusted for the execution environment that is specified in the -backend command line parameter. So far, the available options are 'local', 'pbs' or 'grid' and are explained in the 'usage' section. Since the distribution and availability of computation resources varies among different HPC environments, it tries to maximize the utilization of resources. Moreover, MOLGENIScompute handles iterations in the pipeline (for example, for each chromosome), orders the scripts in the correct order and generates a submission script, named submit. sh that, when executed, submits the complete pipeline to the user-defined HPC environment [21]. All these orchestration actions take place without needing any user interaction.

Finally, we have wrapped the user interaction with the pipeline within a single python script with simple command line arguments. However, all the generated scripts as well as the tool output are still accessible for inspection and review, if needed. To ease installation and usage we have wrapped all the essential operations in a molgenisimpute.py python script that automatically installs all the necessary components on 'setup' (see Table 1) and also eases running of the pipeline (see usage, below). Figure 1 depicts the main architecture and functionality of this python script.

\section{Results and discussion}

We first describe the usage of MOLGENIS-impute and then discuss the practical issues, including installation on Amazon EC2 cloud.

\section{Usage}

To install MOLGENIS-impute, clone the following git repository:

$$
\begin{aligned}
& \text { gitclonegit@github.com: molgenis/ } \\
& \text { molgenis - imputation.git }
\end{aligned}
$$

To download and set up all necessary tools, genetic map and example data (Table 1) run:

$$
\text { python molgenis - impute.py - -dl_tools }
$$

The tools and scripts that this command installs are compatible with any modern 64 bit Linux operating system. For a complete list of system and software dependencies, see Additional file 1.

The command to list all reference panels that are either installed or available for download is:

python molgenis - impute.py - -list

So far, the following reference panels are available for download:

GIANT.phase1_release_v3.20101123: This is a reference panel prepared from the GIANT consortium [24]. It contains all 1,092 samples from the 1,000 Genomes Project, excluding monomorphic and singleton sites.

GIANT.metabo.phase1_release_v3.20101123: This is a Metobochip- [25] specific reference panel that focuses on well-imputed, fine-mapped regions.

1,000_Genomes_phase3_build37: This dataset is based on sequence data from 2,504 samples from the 1,000 Genomes Project, phase 3 [26].

The command to download a reference panel is:

$$
\begin{gathered}
\text { python molgenis - impute.py } \\
-- \text { dl_reference }<\text { NAME }>
\end{gathered}
$$

We also include commands to install and use an arbitrary imputation reference panel. After these steps, a user can continue with the following steps, depicted in Fig. 2.

Liftovering (optional step):

$$
\text { python molgenis - impute.py \ }
$$




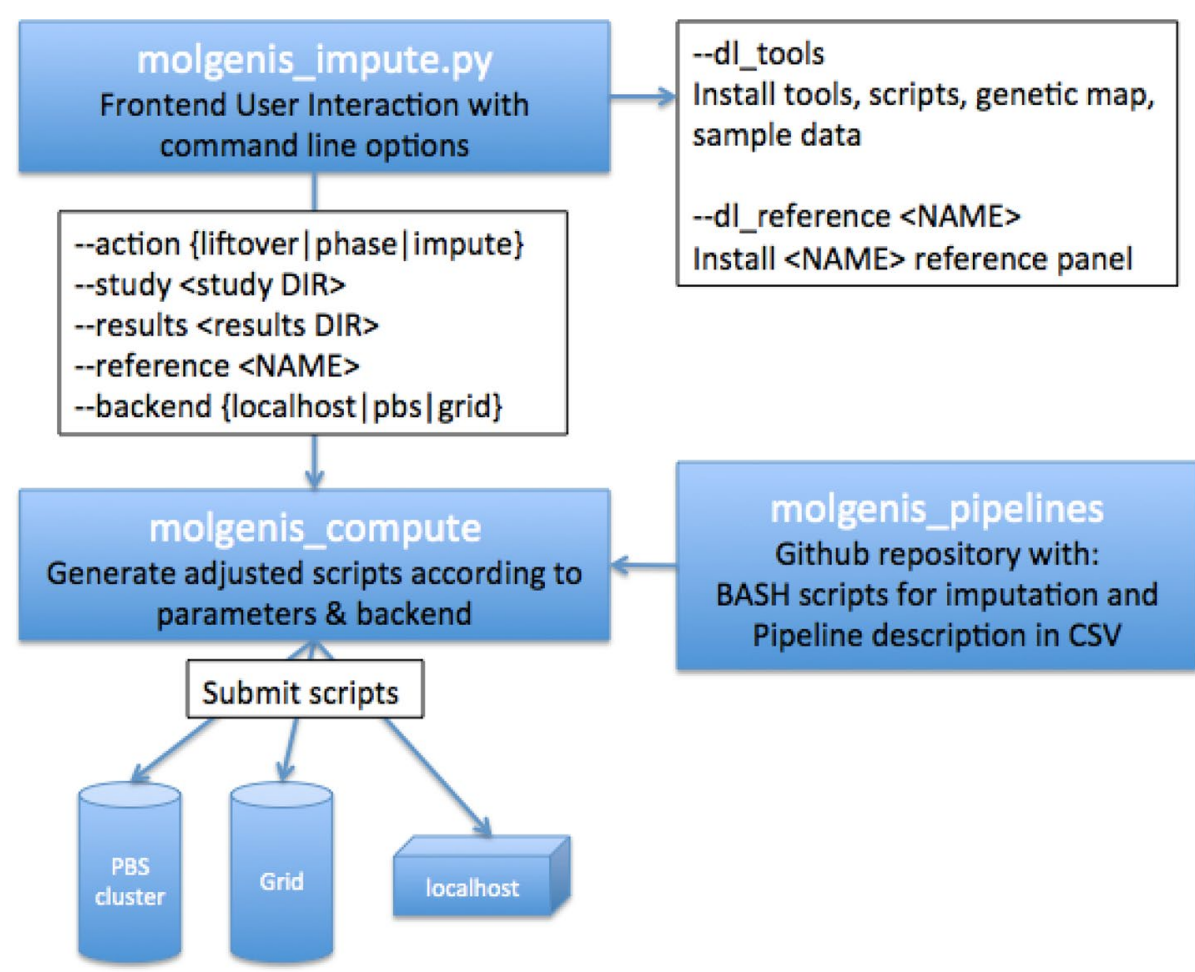

Fig. 1 Outline of MOLGENIS-impute architecture. molgenis_impute.py is the python script with which the user interacts. The script can either install tools and reference panels or use MOLGENIS-compute to create and submit imputation scripts. The imputation BASH scripts and description of the pipeline are in a separate git repository.

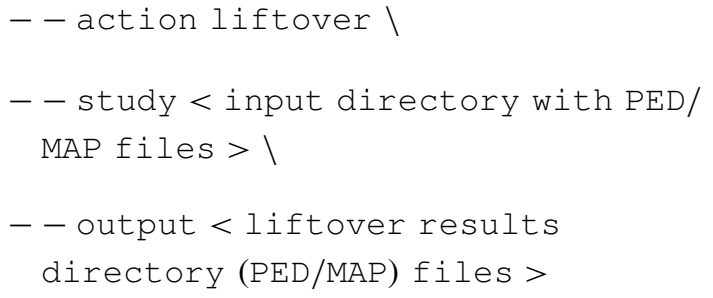

Phasing:

$$
\begin{aligned}
& \text { python molgenis - impute.py } \backslash \\
& \text { - - action phase } \backslash \\
& \text { - - study < liftover results directory } \\
& \text { (PED/MAP) files }>\backslash \\
& \text { - - output < phasing results directory }>
\end{aligned}
$$

Imputing:

$$
\begin{aligned}
& \text { python molgenis - impute.py } \backslash \\
& \text { - - action impute } \backslash
\end{aligned}
$$

\section{-- study $<$ phasing results directory $>1$ \\ - - output < imputation results directory >}

Many additional options exist for refining the presented steps covering all possible options of IMPUTE2 and SHAPEIT2. The options -action liftover_phase_impute and liftover_phase _impute can be used in order to combine the presented steps in a single run. Moreover, liftovering and phasing commands also accept binary PLINK files (BED/BIM/ FAM). Detailed documentation is available on the tool's site. Execution can take place on three different environments, according to the -- backend parameter:

local This is the default option. The scripts are adjusted for a single CPU, 64 bit local computer with a Linux operating system. This option does not generate any special HPC headers and is intended mainly for testing purposes.

pbs Adds headers that allow submission to, for example, Portable Batch System (PBS) [27] or Sun Grid Engine (SGE) clusters.

grid Adds headers that allow the submission to a grid middleware, such as glite-WMS grid scheduler. Resources are managed with Storage Resource 


\section{Input GWAS data}

Optional liftover of variant positions in old genome build to genome build of reference.

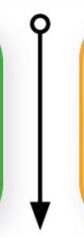

python molgenis-impute.py $\backslash$

--study my_study 1

--output my_study_liftovered \

--action liftover

GWAS data in correct genome build

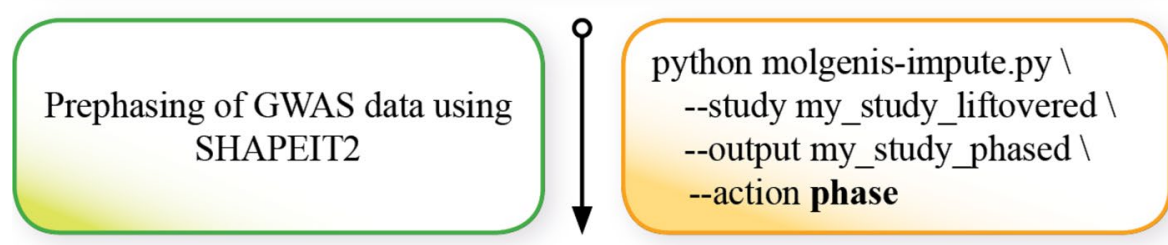

Phased GWAS data

Alignment using Genotype Harmonizer and Imputation using IMPUTE2. Chunked per $5 \mathrm{MB}$ and 500 samples python molgenis-impute.py $\backslash$

--study my_study_phased \

--output my_study_imputed \

--reference GIANT.phase1

--action impute

\section{Imputed GWAS data}

Fig. 2 MOLGENIS-impute's workflow of three steps. Liftovering, phasing and imputation. Rectangles on the left contain a description of each step and on the right a respective demo python command.

Management (SRM) [28] and data transfer and submission is managed with Job Description Language (JDL). In order to achieve execution in all nodes of the grid, we employ the MOLGENIS pilot job solution [29], where workflow deployment (i.e. tool availability) is achieved by reusing the environment modules package [30]. Data transfer and pipeline monitoring are hidden in pilot-jobs.

When running on a cluster or grid environment, the submitted jobs can be monitored, queried and, if necessary, re-submitted. The latter means that if the pipeline crashes during execution, a simple re-submission will resume the execution from the point where it stopped.
Moreover, all output results are saved in temporary files and only after the analysis of each step is successfully completed are the temporary files copied to the expected results location. This ensures that even if a failure happens during the saving of the results files, the user will not end up with erroneous or incomplete files. Re-submission always generates new temporary files. After submission, the user receives information on how to access the temporary files, the job outputs and the submission scripts.

Installing all the required tools takes approximately 2 min on an Amazon EC2 virtual machine instance (t2. 
small) and 30 min to download and convert the GIANT. phase1_release_v3.20101123 version of the 1,000 Genomes Project. After that, the computational time needed for imputation is as published by the authors of the IMPUTE2 tool [9]. Detailed installation and setting up instructions that cover Amazon EC2 and other computing environments can be found in Additional file 1.

\section{Evaluation}

To evaluate the computation requirements of MOLGENIS-impute, we ran the pipeline in all possible instances of Amazing Elastic Compute Cloud. We used the sample study and reference panel presented above, which is included in the tools and datasets that MOLGENIS-impute initially installs. This analysis resulted in 88,650 imputed markers. The results are shown in Table 2 and reveal that phasing requires less than $10 \mathrm{~s}$ for Step 1, $5 \mathrm{~min}$ and $23 \mathrm{~s}$ for step 2, and $6 \mathrm{~min}$ and $40 \mathrm{~s}$ for Step 3. Table 2 also includes a cost estimation given the current Amazon EC2 prices (November 2014). According to IMPUTE2 documentation, when the study panel is prephased (like in our pipeline), the imputation scales linearly with both the number of imputed markers and the number of samples. Hence, the cost presented in Table 2 can be easily extrapolated for larger datasets on various EC2 instances. Our data show that low to medium instance types exhibit optimal cost benefit.

We evaluated MOLGENIS-impute on various Amazon EC2 instances using the presented sample study (100 samples of HapMap2 v. 3, 4,836 markers in chromosome 1) and reference panel (1,092 samples of the GIANT release of the 1,000 Genomes Project reference panel limited to 88,650 SNPs in positions from 1 to 15 million of chromosome 1). All runs used standard parameters. The results show that use of Amazon EC2 low to medium instances is quite cost-effective. According to the authors of IMPUTE2, the imputation scales linearly for number of samples/markers, so the cost can be estimated for larger datasets. ECU is EC2 Compute Unit, a relative measure of the processing power of an EC2 instance.

\section{Applications}

MOLGENIS-impute has been used as the main imputation platform for the Genome of the Netherlands (GoNL) project [31]. GoNL is a whole-genome-sequencing project in a representative population sample, consisting of 250 trio-families from all the provinces in the Netherlands. It aimed to characterize DNA sequence variation in the Dutch population [32]. An initial study assessed the performance of GoNL as a novel reference panel for European samples [33]. Another aim of this project was to provide a population specific imputation panel for various Dutch cohorts in order to improve GWA and meta-analysis studies. Studies that have now been imputed using MOLGENIS-impute include: Dutch Prospective ALS Study [34] with 192 samples, Rotterdam Study [35] with 9,878 samples, Cohort on Diabetes and Atherosclerosis Maastricht (CODAM) [36] with 574 samples, the National Twin Registry, Amsterdam (NTR) [37] study with 1,700 samples, the LifeLines [38] study with 13,707 samples and the Leiden Longevity Study (LLS) [39] with 1,918 samples. These experiments gave us the opportunity to fine-tune our pipeline and we received valuable feedback from a diverse group of bioinformaticians.

\section{Conclusions}

The main deliverable of our approach is a single script that downloads, configures, installs and runs all the tools, data and scripts necessary for genotype imputation. The pipeline management tool that we use 'under the hood' is MOLGENIS-compute, which generates scripts ready for submission to grid, cluster or local computation environments.

MOLGENIS-impute is intended for bioinformaticians and geneticists who want to minimize the time and effort needed to set up and configure an imputation pipeline that includes all the necessary quality check and data management steps. This approach belongs to the family of open bioinformatics solutions suited for HPC environments. As demonstrated, computationally intense solutions need to

Table 2 Time required to perform imputation

\begin{tabular}{|c|c|c|c|c|c|c|}
\hline Instance type & ECUs & vCPUs & Memory (GiB) & Cost/hour & Phasing & Imputation \\
\hline t2.small & Variable & 1 & 1 & $\$ 0.026$ & $5^{\prime} 23^{\prime \prime}$ & $6^{\prime} 40^{\prime \prime}$ \\
\hline t2.medium & Variable & 1 & 2 & $\$ 0.052$ & $4^{\prime} 28^{\prime \prime}$ & $5^{\prime} 56^{\prime \prime}$ \\
\hline m3.medium & 3 & 1 & 3.75 & $\$ 0.070$ & $3^{\prime} 50^{\prime \prime}$ & $5^{\prime} 50^{\prime \prime}$ \\
\hline m3.large & 6.5 & 2 & 7.5 & $\$ 0.140$ & $3^{\prime} 16^{\prime \prime}$ & $5^{\prime} 33^{\prime}$ \\
\hline m3.xlarge & 13 & 4 & 30 & $\$ 0.280$ & $1^{\prime} 0^{\prime \prime}$ & $5^{\prime} 26^{\prime \prime}$ \\
\hline c3.large & 7 & 2 & 3.75 & $\$ 0.105$ & $3^{\prime} 4^{\prime \prime}$ & $5^{\prime} 11^{\prime \prime}$ \\
\hline c3.xlarge & 14 & 4 & 7.5 & $\$ 0.210$ & $1^{\prime} 35^{\prime \prime}$ & $5^{\prime} 6^{\prime \prime}$ \\
\hline c3.2xlarge & 28 & 8 & 15 & $\$ 0.420$ & $0^{\prime} 56^{\prime \prime}$ & $5^{\prime} 5^{\prime \prime}$ \\
\hline
\end{tabular}


have gateways for environments like the cloud [40,41] and grid [42] in order to be directly executable.

No special set up for the execution or programming language knowledge is required. The format for parameters and workflows is CSV. Simplicity and expandability was a primary development goal [43]. In this way, MOLGENIS-impute can easily act as a component of more complex genetic pipelines.

Currently, the presented pipeline supports a subset of available imputation software. Our priority was to offer a tightly coupled and tested pipeline that utilizes wellknown tools. Nevertheless, additional tools like BEAGLE and $\mathrm{MaCH} / \mathrm{Minimac}$ can expand the functionality and cover more uses. Adding and editing tools or computational steps in the pipeline is straightforward for a bioinformatician and is covered in the online documentation of MOLGENIS-compute [44]. Some additional effort is needed in order to adapt the presented python wrapper to these potential additions. Easing modifications in the python wrapper and extending the list of computational environments of MOLGENIS-compute is one of our future aims. More importantly, we plan to upgrade the pipeline when new imputation best practices appear.

\section{Availability and requirements}

The source code, documentation, installation instructions and requirements are available in the following github repository:

https://github.com/molgenis/molgenis-imputation.

License: Simplified BSD License.

\section{Additional files}

Additional file 1: MOLGENIS-impute system requirements.

Additional file 2: Computation of IMPUTE2's info metric.

\begin{abstract}
Abbreviations
CSV: comma separated values; ECU: EC2 compute unite; GWAS: genome-wide association studies; HPC: high performance computing; LD: linkage disequilibrium; NGS: next generation sequencing; PBS: portable batch system; SNP: single nucleotide polymorphism.
\end{abstract}

\section{Authors' contributions}

AK developed the python script, contributed to the imputation pipeline, and tested it on the Lifelines dataset. PD contributed on the BASH pipeline and built the tools for quality control. FvD applied and tested the tool in Rotterdam and CODAM and fine-tuned it. HB and MD developed MOLGENIS-compute and built the submission mechanisms for the PBS and grid environment. MS conceived the idea and designed the main functionality. All authors read and approved the final manuscript.

\section{Acknowledgements}

We thank Jackie Senior for editing the manuscript and Matthijs H. Moed, Leiden University Medical Center, for testing the software.

\section{Funding}

The research leading to these results has received funding from the Ubbo Emmius fund to AK and MS, and from BBMRI-NL, a research infrastructure financed by the Netherlands Organization for Scientific Research (NWO project 184.021.007), to MS, PD and FvD.

Ethics

No new data was generated for this study, so no ethical approval was required.

\section{Compliance with ethical guidelines}

\section{Competing interests}

The authors declare that they have no competing interests.

Received: 7 July 2014 Accepted: 30 July 2015

Published online: 19 August 2015

\section{References}

1. Marchini J, Howie B, Myers S, McVean G, Donnelly P (2007) A new multipoint method for genome-wide association studies by imputation of genotypes. Nat Genet 39(7):906-913

2. Howie BN, Donnelly P, Marchini J (2009) A flexible and accurate genotype imputation method for the next generation of genome-wide association studies. PLoS Genet 5(6):e1000529

3. Lu JT, Wang Y, Gibbs RA, Yu F (2012) Characterizing linkage disequilibrium and evaluating imputation power of human genomic insertion-deletion polymorphisms. Genome Biol 13(2):R15

4. Holm H, Gudbjartsson DF, Sulem P, Masson G, Helgadottir HT, Zanon C et al (2011) A rare variant in MYH6 is associated with high risk of sick sinus syndrome. Nat Genet 43(4):316-320

5. Browning BL, Browning SR (2009) A unified approach to genotype imputation and haplotype-phase inference for large data sets of trios and unrelated individuals. Am J Hum Genet 84(2):210-223

6. Uh HW, Deelen J, Beekman M, Helmer Q, Rivadeneira F, Hottenga JJ et al (2012) How to deal with the early GWAS data when imputing and combining different arrays is necessary. Eur J Hum Genet 20(5):572-576

7. International Parkinson Disease Genomics Consortium, Nalls MA, Plagnol V, Hernandez DG, Sharma M, Sheerin UM et al (2011) Imputation of sequence variants for identification of genetic risks for Parkinson's disease: a meta-analysis of genome-wide association studies. Lancet 377(9766):641-649

8. Johansen TC, Wang J, Lanktree BM, Cao H, McIntyre DA, Ban RM et al (2010) Excess of rare variants in genes identified by genome-wide association study of hypertri-glyceridemia. Nat Genet 42(8):684-687

9. Howie B, Fuchsberger C, Stephens M, Marchini J, Abecasis GR (2012) Fast and accurate genotype imputation in genome-wide association studies through pre-phasing. Nat Genet 44(8):955-959

10. Hao K, Chudin E, McElwee J, Schadt EE (2009) Accuracy of genome-wide imputation of untyped markers and impacts on statistical power for association studies. BMC Genet 10:27

11. Marchini J, Howie B (2010) Genotype imputation for genome-wide association studies. Nat Rev Genet 11(7):499-511

12. Nho K, Shen L, Kim S, Swaminathan S, Risacher SL, Saykin AJ et al (2011) The effect of reference panels and software tools on genotype imputation. In: Proceedings of the Annual AMIA Symposium: 22-26 October 2011. AMIA, Washington DC, pp 1013-1018

13. Pei YF, Li J, Zhang L, Papasian CJ, Deng HW (2008) Analyses and comparison of accuracy of different genotype imputation methods. PLoS One 3(10):e3551

14. Hancock DB, Levy LJ, Gaddis CN, Bierut JL, Saccone LN, Page PG et al (2012) Assessment of genotype imputation performance using 1,000 Genomes in African American studies. PLoS One 7(11):e50610

15. Laughbaum A (2013) Comparing BEAGLE, IMPUTE2, and Minimac Imputation methods for accuracy, computation time, and memory usage. http://blog.goldenhelix.com/?p=1911. Accessed 11 Aug 2015

16. O'Connell J, Gurdasani D, Delaneau O, Pirastu N, Ulivi S, Cocca M et al (2014) A general approach for haplotype phasing across the full spectrum of relatedness. PLoS Genet 10(4):e1004234

17. Goecks J, Nekrutenko A, Taylor J, Galaxy Team (2010) Galaxy: a comprehensive approach for supporting accessible, reproducible, and 
transparent computational research in the life sciences. Genome Biol 11(8):R86

18. Wolstencroft K, Haines R, Fellows D, Williams A, Withers D, Owen S et al (2013) The Taverna workflow suite: designing and executing workflows of Web Services on the desktop, web or in the cloud. Nucleic Acids Res 41(Web Server issue):W557-W561

19. Orvis J, Crabtree J, Galens K, Gussman A, Inman JM, Lee E et al (2010) Ergatis: a web interface and scalable software system for bioinformatics workflows. Bioinformatics 26(12):1488-1492

20. Estrada K, Abuseiris A, Grosveld FG, Uitterlinden AG, Knoch TA, Rivadeneira F (2009) GRIMP: a web- and grid-based tool for high-speed analysis of large-scale genome-wide association using imputed data. Bioinformatics 25(20):2750-2752

21. Byelas H, Kanterakis A, Swertz MA (2013) Towards a Molgenis-based computational framework. In: Kilpatrick P, Milligan P, Stotzka R (eds) Proceedings of IEEE 19th EUROMICRO International Conference on Parallel, Distributed and Network-Based Computing: 27 Feb-1 Mar 2013. CPS, Belfast UK, pp 331-339

22. Purcell S, Neale B, Todd-Brown K, Thomas L, Ferreira MA, Bender D et al (2007) PLINK: a tool set for whole-genome association and populationbased linkage analyses. Am J Hum Genet 81(3):559-575

23. Deelen $\mathrm{P}$, Bonder MJ, van der Velde KJ, Westra H-J, Winder $\mathrm{E}$, Hendriksen D et al (2014) Genotype harmonizer: automatic strand alignment and format conversion for genotype data integration. BMC Res Notes 7:901

24. GIANT consortium (2015) http://www.broadinstitute.org/collaboration/ giant/index.php/GIANT_consortium. Accessed 11 Aug 2015

25. Voight BF, Kang HM, Ding J, Palmer CD, Sidore C, Chines PS et al (2012) The metabochip, a custom genotyping array for genetic studies of metabolic, cardiovascular, and anthropometric traits. PLoS Genet 8(8):e1002793

26. Howie BN, Donnelly P, Marchini J (2014) 1,000 Genomes haplotypesPhase 3 integrated variant set release in NCBI build 37 (hg19) coordinates. http://mathgen.stats.ox.ac.uk/impute/1000GP\%20Phase\%203\%20haplotypes\%206\%200ctober\%202014.html. Accessed 11 Aug 2015

27. Staples G (2006) TORQUE resource manager. In: Proceedings of the 2006 ACM/IEEE conference on Supercomputing. 11-17 November 2006. ACM, Tampa, p 8

28. Nabrzyski J, Schopf JM, Węglarz J (eds) (2004) Grid Resource Management. International Series in Operations Research \& Management Science, vol 64. Springer US, Boston, MA

29. Byelas H, Swertz MA (2013) Scaling bio-analyses from computational clusters to grids. In: Kiss T (ed) Proceedings of the 5th International Workshop on Science Gateways (IWSG 2013): 3-5 June 2013. Published on CEUR-WS.org, Zurich p 8

30. Furlani JL (1991) Modules: providing a flexible user environment. In: Proceedings of the fifth large installation systems administration conference (LISA V), pp 141-152
31. Francioli CL, Menelaou A, Pulit LS, van Dijk F, Palamara FP, Elbers CC et al (2014) Whole-genome sequence variation, population structure and demographic history of the Dutch population. Nat Genet 46(8):818-825

32. Boomsma DI, Wijmenga C, Slagboom EP, Swertz MA, Karssen LC, Abdellaoui A et al (2014) The Genome of the Netherlands: design, and project goals. Eur J Hum Genet 22(2):221-227

33. Deelen P, Menelaou A, van Leeuwen EM, Kanterakis A, van Dijk F, MedinaGomez C et al (2014) Improved imputation quality of low-frequency and rare variants in European samples using the 'Genome of The Netherlands'. Eur J Hum Genet 22(11):1321-1326

34. de Jong SW, Huisman MH, Sutedja NA, van der Kooi AJ, de Visser M, Schelhaas HJ et al (2012) Smoking, alcohol consumption, and the risk of amyotrophic lateral sclerosis: a population-based study. Am J Epidemiol 176(3):233-239

35. Hofman A, Darwish Murad S, van Duijn CM, Franco OH, Goedegebure A Ikram MA et al (2013) The Rotterdam Study: 2014 objectives and design update. Eur J Epidemiol 28(11):889-926

36. Wlazlo N, van Greevenbroek MM, Ferreira I, Jansen EH, Feskens EJ, van der Kallen CJ et al (2013) Iron metabolism is associated with adipocyte insulin resistance and plasma adiponectin: the Cohort on Diabetes and Atherosclerosis Maastricht (CODAM) study. Diabetes Care 36(2):309-315

37. Boomsma DI, Vink JM, van Beijsterveldt TC, de Geus EJ, Beem AL, Mulder EJ et al (2002) Netherlands twin register: a focus on longitudinal research. Twin Res 5:401-406

38. Stolk RP, Rosmalen JG, Postma DS, de Boer RA, Navis G, Slaets JP et al (2008) Universal risk factors for multifactorial diseases: LifeLines: a threegeneration population-based study. Eur J Epidemiol 23(1):67-74

39. Schoenmaker M, de Craen AJ, de Meijer PH, Beekman M, Blauw GJ, Slagboom PE et al (2006) Evidence of genetic enrichment for exceptional survival using a family approach: the Leiden Longevity Study. Eur J Hum Genet 14(1):79-84

40. Wall DP, Kudtarkar P, Fusaro VA, Pivovarov R, Patil P, Tonellato PJ (2010) Cloud computing for comparative genomics. BMC Bioinform 11:259

41. Schatz MC, Langmead B, Salzberg SL (2010) Cloud computing and the DNA data race. Nat Biotechnol 28(7):691-693

42. Stevens RD, Robinson AJ, Goble CA (2003) myGrid: personalised bioinformatics on the information grid. Bioinformatics 19(Suppl 1):i302-i304

43. Wilson G, Aruliah DA, Brown CT, Chue Hong NP, Davis M, Guy RT et al (2014) Best practices for scientific computing. PLoS Biol 12(1):e1001745

44. Molgenis Compute 5 User Guide (2015) https://rawgit.com/molgenis/ molgenis-compute/master/molgenis-compute-core/README.html. Accessed 11 Aug 2015

\section{Submit your next manuscript to BioMed Central and take full advantage of:}

- Convenient online submission

- Thorough peer review

- No space constraints or color figure charges

- Immediate publication on acceptance

- Inclusion in PubMed, CAS, Scopus and Google Scholar

- Research which is freely available for redistribution

Submit your manuscript at 